\title{
MECHANICAL RESPONSE IN AN $N$-ELECTRODE PIEZO-QUARTZ BAR UNDER ELECTRICAL AND THERMAL EXCITATIONS
}

\author{
T.K. MUNSHI \\ Department of Physics, Kharagpur College \\ Kharagpur, Dist. Midnapore, West Bengal, India \\ K.K. KUNDU \\ Department of Physics, City College, Calcutta 700009, India \\ and R.K. Mahalanabis \\ Department of Mathematics, Jadavpur University, Jadavpur, Calcutta 700032, India \\ (Received July 14, 1994; revised version Oclober 6, 1994; \\ in final form March 20, 1995)
}

\begin{abstract}
The mechanical response in an $N$-electrode piezo-quartz bar where two consecutive electrodes are separated by an unelectroded region has been studied under electrical and thermal excitations. The bar is subjected to a periodic polarization gradient and a constant flow of heat. The method of operational calculus and Green's function has been used to solve the problem. For time scale ranging from 0.1 to $1.0 \mathrm{~s}$ the response becomes a linear function of time and is of the order of $10^{-8} \mathrm{~m}$.

PACS numbers: 77.65. $-\mathrm{j}, 77.70 .+\mathrm{a}$
\end{abstract}

\section{Introduction}

The studies in the disturbances of a piezoelectric material from the stand point of mechanics of continuous media have been initiated by Mason [1], Cady [2], Redwood [3] and later developed by Mindlin [4], Rizzo [5] etc. They considered the piezoelectric problems from the point of view of circuit theory where the situation provides two fields, viz. mechanical and electrical and certainly the studies become more interesting if the above interaction is coupled with a thermal field (Samoilov and Shchedrina [6], Balabaev and Ivina [7], Baranski et al. [8], Munshi et al. [9]). Due to various applications in under-water signaling (Shiosaki and 
Kawabata [10], Imai [11]) the mechanical response of $N$-electrode piezo-quartz bar has been studied by a number of researchers like Holland [12], Ray [13], Nowotny et al. [14] etc. The present study is to investigate the mechanical response of an $N$-electrode piezo-quartz bar in which two consecutive electrodes are separated by an unelectroded region under periodic polarization gradient and a constant flow of heat. Maxwell's equation, the equation of steady state heat flow and the relevant piezoelectric constitutive equations have been used to solve the problem. The variation of the disturbances with time ranging from 0.1 to $1.0 \mathrm{~s}$ is found to be linear in nature and is of the order of $10^{-8} \mathrm{~m}$.

\section{The problem, fundamental equations and boundary conditions}

The problem considered here is an $N$-electrode piezoelectric bar (Fig. 1) such that two consecutive electrodes are separated by an unelectroded region and with the choice of the origin, the coordinate axes and the application of the mechanical force, it is assumed by Nowotny [14] that the cross-sectional dimensions $(\omega, \tau)$ are small so that $T_{1}$ (stress) and $P_{1}$ (electric polarization) are functions of $x$ and $t$ only. Here $\omega$, the height of the electrode, is along $y$-axis and $\tau$, the width of the electrode, is along $z$-axis. The length of the electrode is taken along the $x$-axis.

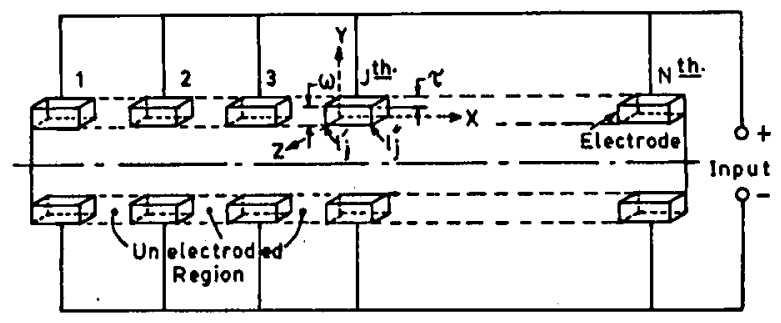

Fig. 1. An $N$-electrode piezo-quartz bar in which two consecutive electrodes are separated by an unelectroded region is under input signals.

We consider the bar to be subjected to a periodic polarization gradient and a constant flow of heat. Our object is to determine the mechanical response that stems from the interaction of electrical and thermal fields. The fundamental equations are therefore the equation of mechanical motion, the equation of electricity and the equation of heat flow. The equation of mechanical motion is given by

$$
\frac{\partial T_{1}}{\partial x}=\rho \frac{\partial^{2} \psi}{\partial t^{2}}
$$

where $T_{1}$ is the stress, $\psi$ - the displacement in the $x$-direction and $\rho$ - the material density.

The piezoelectric equations due to Mindlin [4] are given by

$$
\begin{aligned}
& T_{1}=c_{11} S_{1}+e_{11} E_{1}+\lambda_{1} \theta, \\
& P_{1}=e_{11} S_{1}+K_{11} E_{1}+p_{1} \theta,
\end{aligned}
$$

where $S_{1}$ is the normal strain $(=\partial \psi / \partial x), E_{1}$ - the electric field strength, $P_{1}-$ the electric polarization, $c_{11}$ - the elastic-stress coefficient, $K_{11}$ - the electric 
susceptibility, $e_{11}$ - the piezoelectric stress constant, $\theta-$ the temperature, $p_{1}-$ the pyroelectric constant, $\lambda_{1}$ - the temperature coefficient. The other necessary equation for this problem to complete the set of equations for the three effects is the heat conduction equation

$$
K \frac{\partial^{2} \theta}{\partial x^{2}}=\frac{\partial \theta}{\partial t}
$$

where $K$ is the diffusivity constant and $\theta$ is the temperature.

In accordance with our assumption,

$$
\begin{aligned}
& -P_{1}= \begin{cases}P_{0} / \tau & \text { under electroded region, } \\
0 & \text { under unelectroded region, }\end{cases} \\
& +\theta= \begin{cases}\theta_{0} / \tau & \text { under electroded region, } \\
0 & \text { under unelectroded region, }\end{cases}
\end{aligned}
$$

where $P_{0}$ and $\theta_{0}$ are constants and

$$
\begin{aligned}
& -\frac{\partial P_{1}}{\partial x}=\sum_{j=1}^{N} \frac{P_{0}}{\tau}\left[\delta\left(x-l_{j}^{1}\right)-\delta\left(x-l_{j}^{11}\right)\right] \sin \alpha t \\
& +\frac{\partial \theta}{\partial x}=\sum_{j=1}^{N} \frac{\theta_{0}}{\tau}\left[\delta\left(x-l_{j}^{1}\right)-\delta\left(x-l_{j}^{11}\right)\right],
\end{aligned}
$$

where $\alpha$ is constant and $l_{j}^{1}$ and $l_{j}^{11}$ denote length of the left and right side of the $j$-th electrode respectively.

\section{Methods and solution}

From Eqs. (1a), (1b) with the aid of equation of motion we get

$$
\frac{\partial^{2} \psi}{\partial x^{2}}\left(c_{11}-\frac{e_{11}^{2}}{K_{11}}\right)-\rho \frac{\partial^{2} \psi}{\partial t^{2}}=-\left(\lambda_{1}-\frac{e_{11} p_{1}}{K_{11}}\right) \frac{\partial \theta}{\partial x}-\frac{e_{11}}{K_{11}} \frac{\partial P_{1}}{\partial x} .
$$

Now applying Laplace transform of parameter $p$ to Eq. (4), we obtain from Eqs. (4), (3a) and (3b):

$$
\begin{aligned}
\frac{\partial^{2} \psi}{\partial x^{2}} & +\frac{\rho p^{2} \psi}{e_{11}^{2} / K_{11}-c_{11}}=\frac{\lambda_{1}-e_{11} p_{1} / K_{11}}{e_{11}^{2} / K_{11}-c_{11}} \frac{\overline{\partial \theta}}{\partial x} \\
& +\frac{e_{11}}{K_{11}\left(e_{11}^{2} / K_{11}-c_{11}\right)} \frac{\overline{\partial P_{1}}}{\partial x}
\end{aligned}
$$

where

$$
\begin{aligned}
& -\frac{\overline{\frac{\partial P_{1}}{\partial x}}}{\partial}=\frac{\alpha}{p^{2}+\alpha^{2}} \sum_{j=1}^{N} \frac{P_{0}}{\tau}\left[\delta\left(x-l_{j}^{1}\right)-\delta\left(x-l_{j}^{11}\right)\right], \\
& +\frac{\overline{\partial \theta}}{\partial x}=\frac{1}{p} \sum_{j=1}^{N} \frac{\theta_{0}}{\tau}\left[\delta\left(x-l_{j}^{1}\right)-\delta\left(x-l_{j}^{11}\right)\right] .
\end{aligned}
$$

It will be convenient to integrate Eq. (5) utilizing Green's function (Munshi et al. [15]). 
As in Holland [12], Ray [13] let a force of value $\omega \tau\left(c_{11}-e_{11}^{2} / K_{11}\right)$ be applied to the bar in the $x$-direction at some point $x_{2}$. Let the bar have no voltage and no other forces applied and let the ends be free. We define Green's function evaluated at some other point $x_{1}$ to be the resulting particle displacement at the point.

Mathematically, this means Green's function $G\left(x_{1} / x_{2}\right)$ obeys the differential equation

$$
\left(\frac{\partial^{2}}{\partial x_{1}^{2}}+\frac{\rho p^{2}}{e_{11}^{2} / K_{11}-c_{11}}\right) G\left(x_{1} / x_{2}\right)=-\delta\left(x_{1}-x_{2}\right)
$$

with the boundary conditions

$$
\begin{aligned}
& 0=\frac{T_{1}}{c_{11}-e_{11}^{2} / K_{11}}+\frac{e_{11} P_{1}}{K_{11}\left(e_{11}^{2} / K_{11}-c_{11}\right)}+\left.\frac{\left(\lambda_{1}-p_{1} e_{11} / K_{11}\right) \theta}{e_{11}^{2} / K_{11}-c_{11}}\right|_{x_{1}=0, l} \\
& \quad=\left.S_{1}\right|_{x_{1}=0, l}=\left.\frac{\partial}{\partial x_{1}} G\left(x_{1} / x_{2}\right)\right|_{x_{1}=0, l} .
\end{aligned}
$$

A Fourier expansion of solution for $G\left(x_{1} / x_{2}\right)$ may be found quite easily. Let us assume

$$
G\left(x_{1} / x_{2}\right)=A_{0}\left(x_{2}\right)+\sum_{n=1}^{\infty} A_{n}\left(x_{2}\right) \cos \frac{n \pi x_{1}}{l} .
$$

Therefore,

$$
G\left(x_{1} / x_{2}\right)=\frac{1}{p l p^{2} /\left(e_{11}^{2} / K_{11}-c_{11}\right)}-\sum_{n=1}^{\infty} \frac{\frac{2}{l} \cos \left(n \pi x_{1} / l\right) \cos \left(n \pi x_{2} / l\right)}{\frac{p^{2} \rho}{e_{11}^{2} / K_{11}-c_{1}}-\frac{n^{2} \pi^{2}}{l^{2}}}
$$

Here, we note that $G\left(x_{1} / x_{2}\right)$ is symmetric in $x_{1}$ and $x_{2}$. In spite of the previous definition of $x_{1}$ and $x_{2}$, we shall have $x_{1}$ the source coordinate and $x_{2}$ - the observer coordinate. The convention makes subsequent results more convenient.

The wave equation can be integrated for $\bar{\psi}_{1}$. Multiplying Eq. (7) by $\bar{\psi}_{1}\left(x_{1}\right)$ and Eq. (5) by $G\left(x_{1} / x_{2}\right)$ and subtracting second one from first one, let us integrate the result from $\varepsilon$ to $l-\varepsilon$, where $\varepsilon$ is an infinitesimal quantity as follows:

$$
\begin{aligned}
& \int_{\varepsilon}^{l-\varepsilon}\left\{\bar{\psi}_{1}\left(x_{1}\right)\left[\frac{\partial^{2} G\left(x_{1} / x_{2}\right)}{\partial x_{1}^{2}}+\frac{\rho p^{2}}{e_{11}^{2} / K_{11}-c_{11}} G\left(x_{1} / x_{2}\right)\right]\right. \\
& \left.-G\left(x_{1} / x_{2}\right)\left[\frac{\partial^{2} \bar{\psi}_{1}\left(x_{1}\right)}{\partial x_{1}^{2}}+\frac{\rho p^{2} \bar{\psi}_{1}\left(x_{1}\right)}{e_{11}^{2} / K_{11}-c_{11}}\right]\right\} \mathrm{d} x_{1} \\
& =\int_{\varepsilon}^{l-\varepsilon}\left\{\bar{\psi}_{1}\left(x_{1}\right)\left[-\delta\left(x_{1}-x_{2}\right)\right]-G\left(x_{1} / x_{2}\right)\right. \\
& \left.\times\left[\frac{\lambda_{1}-e_{11} p_{11} / K_{11}}{e_{11}^{2} / K_{11}-c_{11}} \frac{\partial \bar{\theta}}{\partial x_{1}}+\frac{e_{11}}{K_{11}\left(e_{11}^{2} / K_{11}-c_{11}\right)} \frac{\partial P_{1}}{\partial x_{1}}\right]\right\} \mathrm{d} x_{1},
\end{aligned}
$$




$$
\begin{aligned}
& \bar{\psi}_{1}\left(x_{2}\right)=-\left[\bar{\psi}_{1}\left(x_{1}\right) \frac{\partial G\left(x_{1} / x_{2}\right)}{\partial x_{1}}-G\left(x_{1} / x_{2}\right) \frac{\partial \bar{\psi}_{1}\left(x_{1}\right)}{\partial x_{1}}\right]_{\varepsilon}^{l-\varepsilon} \\
& -\int_{\varepsilon}^{l-\varepsilon} G\left(x_{1} / x_{2}\right)\left[\frac{\lambda_{1}-e_{11} p_{1} / K_{11}}{e_{11}^{2} / K_{11}-c_{11}} \frac{\partial \bar{\theta}}{\partial x_{1}}+\frac{e_{11}}{K_{11}\left(e_{11}^{2} / K_{11}-c_{11}\right)} \frac{\partial \bar{P}_{1}}{\partial x_{1}}\right] \mathrm{d} x_{1} .
\end{aligned}
$$

For the boundary condition in Eq. (7), the first term within the bracket in Eq. (10), i.e. $\bar{\psi}_{1}\left(x_{1}\right) \partial G\left(x_{1} / x_{2}\right) / \partial x_{1}$ vanishes. The second term in Eq. (10) may be expressed as

$$
\left.G\left(x_{1} / x_{2}\right) \frac{\partial \bar{\psi}_{1}\left(x_{1}\right)}{\partial x_{1}}\right|_{\varepsilon} ^{l-\varepsilon}=\left.G\left(x_{1} / x_{2}\right) \bar{S}_{1}\right|_{\varepsilon} ^{l-\varepsilon} .
$$

It can be shown that

$$
G\left(x_{1} / x_{2}\right) \bar{S}_{\varepsilon}^{l-\varepsilon}=1 / \omega \tau\left(c_{11}-e_{11}^{2} / K_{11}\right)\left[F_{l} G\left(l / x_{2}\right)-F_{0} G\left(0 / x_{2}\right)\right],
$$

where $F_{l}$ and $F_{0}$ denotes the force at $x=l$ and $x=0$ respectively.

$$
\begin{aligned}
& \bar{\psi}_{1}\left(x_{2}\right)=\frac{1}{\omega \tau \rho l} \frac{F_{1}-F_{0}}{p^{2}}+\frac{2}{\omega \tau \rho l} \sum_{n=1}^{\infty} \frac{\left[(-1)^{n} F_{1}-F_{0}\right] \cos \left(n \pi x_{2} / l\right)}{p^{2}-n^{2} \pi^{2}\left(e_{11}^{2} / K_{11}-c_{11}\right) / \rho l^{2}} \\
& +\frac{2 e_{11} P_{0}}{l \tau \rho K_{11}} \frac{\alpha}{p^{2}+\alpha^{2}} \sum_{j=1}^{N} \sum_{n=1}^{\infty} \frac{\left[\cos \left(n \pi l_{j}^{11} / l\right)-\cos \left(n \pi l_{j}^{1} / l\right)\right] \cos \left(n \pi x_{2} / l\right)}{P^{2}-n^{2} \pi^{2}\left(e_{11}^{2} / K_{11}-c_{11}\right) / \rho l^{2}} \\
& -\frac{2\left(\lambda_{1}-e_{11} P_{1} / K_{11}\right)}{l \tau \rho} \theta_{0} \frac{1}{P} \\
& \quad \times \sum_{j=1}^{N} \sum_{n=1}^{\infty} \frac{\left[\cos \left(n \pi l_{j}^{11} / l\right)-\cos \left(n \pi l_{j}^{1} / l\right)\right] \cos \left(n \pi x_{2} / l\right)}{p^{2}-n^{2} \pi^{2}\left(e_{11}^{2} / K_{11}-c_{11}\right) / \rho l^{2}} .
\end{aligned}
$$

In the case of piezo-quartz, $e_{11}^{2} / K_{11}-c_{11}<0$ :

$$
\begin{aligned}
& \psi_{1}\left(x_{1}\right)=\frac{1}{\omega \tau \rho l}\left(F_{l}-F_{0}\right) t+\frac{2}{\omega \tau \rho l} \sum_{n=1}^{\infty}\left[F_{l}(-1)^{n}-F_{0}\right] \cos \left(n \pi x_{2} / l\right) \sin \beta t / \beta \\
& +\frac{2 e_{11} P_{0}}{l \tau \rho K_{11}} \sum_{j=1}^{N} \sum_{n=1}^{\infty}\left[\cos \left(n \pi l_{j}^{11} / l\right)-\cos \left(n \pi l_{j}^{1} / l\right)\right] \cos \left(n \pi x_{2} / l\right) \\
& \quad \times \frac{1}{\beta\left(\alpha^{2}-\beta^{2}\right)}(\alpha \sin \beta t-\beta \sin \alpha t) \\
& -\frac{2\left(\lambda_{1}-e_{11} p_{1} / K_{11}\right) \theta_{0}}{\rho l \tau} \sum_{j=1}^{N} \sum_{n=1}^{\infty}\left[\cos \left(n \pi l_{j}^{11} / l\right)-\cos \left(n \pi l_{j}^{1} / l\right)\right] \\
& \quad \times \cos \left(n \pi x_{2} / l\right)\left(\mathrm{e}^{-\alpha t}-\cos \beta t+\sin \beta t\right) /\left(\alpha^{2}+\beta^{2}\right),
\end{aligned}
$$

where $\beta^{2}=n^{2} \pi^{2}\left(e_{11}^{2} / K_{11}-c_{11}\right) / \rho l^{2}$. 


\section{Discussion}

Equation (12) represents the mechanical response in an $N$-electrode piezoelectric quartz bar under electrical and thermal excitations.

For numerical calculations, the values of the material constants have been taken from Cady [2], Sasaki and Takeuchi [16], Gibbs [17] etc. while values like $\omega, \tau, l, N, P_{0}, \theta_{0}, \alpha$ and $x_{2}$ have been chosen to facilitate numerical calculation as follows:

$$
\begin{array}{lll}
\omega=0.01 \mathrm{~m}, & N=100, & \theta_{0}=300 \mathrm{~K}, \\
\tau=0.01 \mathrm{~m}, & \alpha=100 \mathrm{~s}^{-1}, & x_{2}=0.2 \mathrm{~m}, 0.3 \mathrm{~m}, 0.4 \mathrm{~m}, \\
l=0.04 \mathrm{~m}, & P_{0}=200 \mathrm{C} / \mathrm{m} . &
\end{array}
$$

In the material piezo-quartz, where $e_{11}^{2} / K_{11}-c_{11}<0$, the mechanical response exhibited by Eq. (12) involves linear, transient and periodic function of time together with a large number of material constants. When $e_{11}^{2} / K_{11}-c_{11}>0$, the mechanical response of $N$-electrode piezoelectric bar under periodic polarization gradient and a constant flow of heat is found to be partly linear, partly transient and partly hyperbolic function of time $t$.

In Eq. (12), the contribution of other terms is insignificant due to the involvement of the term $\beta$ compared to the first term. As a result the disturbance exhibits a linear relationship with time (Fig. 2) and is of the order of $10^{-8} \mathrm{~m}$.

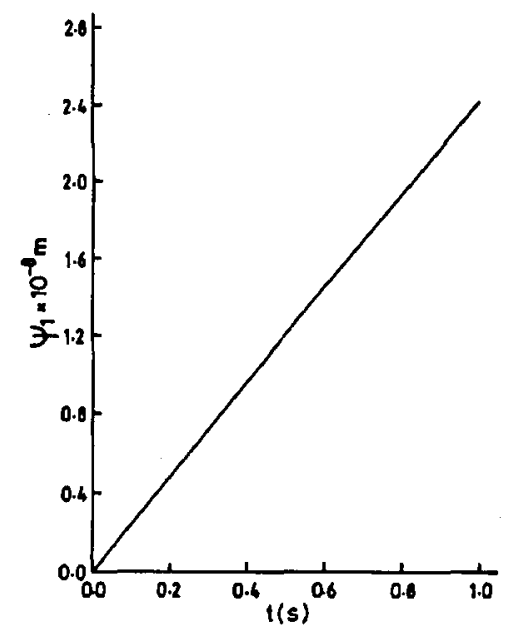

Fig. 2. Variation of the mechanical disturbances in an $N$-electrode piezo-quartz bar with time for constant value of $x_{2}(=0.4 \mathrm{~m})$.

As in Holland [12], Ray [13] let a force of value $\omega \tau\left(c_{11}-e_{11}^{2} / K_{11}\right)$ be applied to the bar in the $x$-direction at some point $x_{2}$. Let the bar have no voltage and no other forces applied and let the ends be free. We define Green's function evaluated at some other point $x_{1}$ to be the resulting particle displacement at the point.

$G\left(x_{1} / x_{2}\right)$ is symmetric in $x_{1}$ and $x_{2}$. In spite of previous definition of $x_{1}$ and 
$x_{2}$ we shall have $x_{1}$ the source coordinate and $x_{2}$ - the observer coordinate to make results more convenient.

It is interesting to note that the expression given out by Eq. (12) yields almost the same disturbances for different values of $x_{2}$ viz. $0.2 \mathrm{~m}, 0.3 \mathrm{~m}$, and $0.4 \mathrm{~m}$. The variation of the disturbances with time for $x_{2}=0.4 \mathrm{~m}$ is shown in Table.

\section{TABLE}

Numerical values of the mechanical disturbance in an $N$-electrode piezo-quartz bar with time for $x_{2}=0.4 \mathrm{~m}$.

\begin{tabular}{c|c}
\hline \hline$t[\mathrm{~s}]$ & $\psi_{1}\left[\times 10^{-8} \mathrm{~m}\right]$ \\
\hline 0.0 & 0.0 \\
0.1 & 0.24 \\
0.2 & 0.48 \\
0.3 & 0.72 \\
0.4 & 0.96 \\
0.5 & 1.20 \\
0.6 & 1.44 \\
0.7 & 1.68 \\
0.8 & 1.92 \\
0.9 & 2.16 \\
1.0 & 2.40
\end{tabular}

\section{Acknowledgment}

The work was performed under UGC-DSA project at the Department of Mathematics, Jadavpur University, Calcutta-32, India.

\section{References}

[1] W.P. Mason, Piezoelectric Crystals and their Applications to Ultrasonics, D. Van Nostrand Co., New York 1950, p. 84.

[2] W.G. Cady, Piezoelectricity, McGraw-Hill, New York 1959, p. 187.

[3] M. Redwood, J. Acoust. Soc. Am. 33, 527 (1961).

[4] R.D. Mindlin, On the Equations of Motion of Piezoelectric Crystals: Problem of Continuum Mechanics, Muskhelishvili Anniversary Vol., S.I.A.M., Philadelphia, Penssylvania 1961, p. 282.

[5] R.R. Rizzo, Ph.D. Dissertation, Univ. Delware, 1966.

[6] V.B. Samoilov, L.V. Shchedrina, Ferroelectrics 118, 225 (1991).

[7] M.S. Balabaev, F.N. Ivina, Sov. Phys.-Acoust. 34, 90 (1988). 
[8] K.N. Baranski, M.A. Zubareva, I.A. Yakolov, JETP Lett. 47, 291 (1988).

[9] T.K. Munshi, K.K. Kundu, R.K. Mahalanabis, Acta Phys. Pol. A 85, 585 (1994).

[10] T. Shiosaki, A. Kawabata, Ferroelectrics 95, 9 (1989).

[11] N. Imai, J. Acoust. Soc. Jpn. 11, 327 (1990).

[12] R. Holland, Proc. Inst. Elec. Electr. Eng. 54, 968 (1966).

[13] D.R. Ray, Proc. N.I.S.I. A 35, 845 (1969).

[14] H. Nowotny, E. Benes, M. Schmid, J. Acoust. Soc. Am. 90, 1238 (1991).

[15] T.K. Munshi, K.K. Kundu, R.K. Mahalanabis, J. Acoust. Soc. Am. 96, 2836 (1994).

[16] Y. Sasaki, Y. Takeuchi, J. Acoust. Soc. Jpn. 10, 181 (1989).

[17] D.F. Gibbs, Ferroelectrics 82, 133 (1988). 